\title{
Application of a Low Frequency, Low Power Radio Frequency Positioning System for Real Time Indoor Positioning
}

\author{
Pooneh Mohaghegh*, Denis Prvica**,Juan Sebastián Botero-Valencia***, Alexis Boegli*, Yves Perriard*+ \\ *Integrated Actuators Laboratory (LAI) \\ Ecole polytechnique federal de Lausanne (EPFL), Neuchatel, Switzerland \\ *+ IEEE Senior Member \\ ** e-liberty (SA), Neuchatel, Switzerland \\ *** Instituto Tecnológico Metropolitano (ITM), Colombia \\ contact: pooneh.mohaghegh@epfl.ch
}

\begin{abstract}
This paper focuses on the design and application of low power and low-frequency localization system using received signal strength indication (RSSI). The receiver will wake up from sleeping mode by a modulated magnetic field emitted from a transmitter module. Then it will send messages containing the Received Signal Strength Indication (RSSI) via WiFi with Message Quest Telemetry Transport (MQTT) protocol to an MQTT broker. Using the magnetic coupling effect and RSSI ranging, the system is a unique candidate for low power security-positioning purposes.

Index Terms-Radio Frequency (RF), low power, magnetic coupling, Received Signal Strength Indication (RSSI), Positioning Systems (PS), Message Quest Telemetry Transport (MQTT)
\end{abstract}

\section{INTRODUCTION}

With ever-growing applications of wireless internet access and Bluetooth, the ubiquity of information and communication network is inevitable. This reflects the penetration of the Internet of Things (IoT) in daily aspects of life [1]. In the network of connecting devices, knowledge of the exact position of each node in the network is critical. [1]

In the majority of positioning systems, an estimate of the unknown position is based on range measurements between transmitters and a receiver. These values are obtained by extracting the information contained in the received signal, such as Received Signal Strength Indication (RSSI) or Time of Arrival (ToA) systems, etc. Received Signal Strength (RSS) as a non-timing base ranging system, does not contain a common offset (or bias). Even though the relationship between the RSSI value and distance for far-field applications is well known, near field antennas barely studied for RSSI based localization. Recently, the advent of electrically small antennas for low-frequency applications, made the research feasible for developing positioning systems based on this property [2], [3].

RFID is an automatic identification process that uses RF wireless communication technology based on electromagnetic transmission between RFID readers and RFID tags for tracking purposes [4], [5]. The RFID tags are classified as passive and active. An active RFID tag that is powered by a battery, that will usually provide a relatively wide transmission range. While the passive tag has a usually a shorter range and it responds to the reader signal after being activated by one. This system is capable of tracking and determining the position of the tag via radio waves [6]. Nowadays, the passive tags are part of daily lives that are used in many bank cards or road toll tags and in portals for monitoring the cargo goods.

We are introducing a new low-frequency active RFID system that suits complex and semi-enclosed environment positioning. Benefiting from $125 \mathrm{kHz}$, this tag enables new applications. This low frequency has substantial advantages such as enhanced diffraction around environmental clutters (i.e., corners), less vulnerability to multi-path confusion, superior penetration depth, and low probability of intercept (LPI) [7]. As a result, this system is a candidate for short-range positioning purposes with relatively accurate results.

In this paper, we are presenting a low radio frequency (RF) positioning system integrated with WiFi for real-time localization application. First, we will define the principles of the RF positioning system. Latter will explain about the communication protocol. Finally, we will apply the presented system in a real positioning scenario.

\section{THE POSITIONING SYSTEM AND COMMUNICATION}

The system is consists of several receivers (tags) and three $125 \mathrm{kHz}$ coils (transmitter) to generate a modulated magnetic field. This field will wake up an integrated chip (AS3933) on the tags as soon as the tags are in close proximity to the generated field. The tag is integrated with a small three dimensional coils which will be coupled with the generated magnetic field of the transmitter once the chip is active. The tag then starts sending an indication of the strength of it's received signal at each channel (RSSI) of the chip through wifi to an MQTT broker. 
Once the AS3933 chip is awake, the tag will connect to a Mosqitto server with the MQTT protocol to communicate. The latter will send the RSSI to a PC for calculations to determine its position. The sensitivity of the AS3933 chip is to be adjusted due to the application properties. The system is using an area of $80 \mathrm{~cm}$ width, $80 \mathrm{~cm}$ length and $2.5 \mathrm{~m}$ height. 2 Three $125 \mathrm{kHz}$ coils are used as a low-frequency transmitter for each node.

The settings on the receiver side can be adjusted due to the desired range of application, environmental properties of the test area, and power consumption. The damping factor and the gain of the AS3933 can be changed to results in a linear relationship between the RSSI and the distance from coils. The previous publication of our group completely discussed the optimal required settings for the goal of this paper. Hence, we are using a $-12 d B$ gain reduction and $3 k \Omega$ damping factor for the $3 \mathrm{D}$ coil on the tag.

\section{A. THE COMMUNICATION PROTOCOL}

The tag (red box in figure 3) publishes a message to the broker. The data to be processed consists of the RSSI values of three antennas in three positions as described in figure 3. For each antenna in the receiver, we receive three RSSI values in three channels. As a result, for a single position of the tag, we receive 9 values of RSSI.

All the information about the position of the card and its identity, battery state are sent to a docker engine. Docker is a set of the platform as a service (PaaS) [8] products that use OS-level virtualization to deliver software in packages called containers. Containers are isolated from one another and bundle their own software, libraries, and configuration files; they can communicate with each other through well-defined channels. All containers are run by a single operating-system kernel and are thus more lightweight than virtual machines.

Using PostgreSQL [9] as an open-source objectrelational database system, provide a reliable environment to safely store and scale the complicated data workloads. PostgreSQL has earned a strong reputation for its proven architecture, reliability, data integrity, robust feature set, extensively, and the dedication of the open-source community behind the software to consistently deliver performance and innovative solutions.

We are using Eclipse Mosquitto [10] which is a lightweight open-source (EPL/EDL licensed) message broker that implements the MQTT protocol versions 5.0, 3.1.1 and 3.1. Mosquitto is suitable for use on all devices from low power single board computers to full servers. The MQTT protocol provides a lightweight method of carrying out messaging using a publish/subscribe model. This makes it suitable for Internet of Things messaging especially for embedded computers or microprocessors with low power consumption. Mosiquitto also provides a C library for implementing MQTT clients.

Each physical node (tag) in the system consists of a $125 \mathrm{kHz}$ receiver integrated with an ESP32 chip benefiting from a large physical WiFi range and direct connection through a WiFi router while using Bluetooth for convenient broadcast. Using the $125 \mathrm{kHz}$ receiver brings the possibility of sleep and wake-up mode for the system, while the nodes are woken up by a modulated magnetic field of three $125 \mathrm{kHz}$ transmitters 1 . The system enables the possibility of $3 \mathrm{D}$ localization.

Physical Node

$125 \mathrm{kHz}$ Receiver $\mathrm{AS} 3933$
$\mathrm{ESP}_{32}$ 尺

Fig. 1. Schematic of building blocks of each physical node (the receiver) in the network.

By adjusting the settings of transmitter and receiver for the desired range, an empirical relationship between the RSSI value and the distance was extracted.

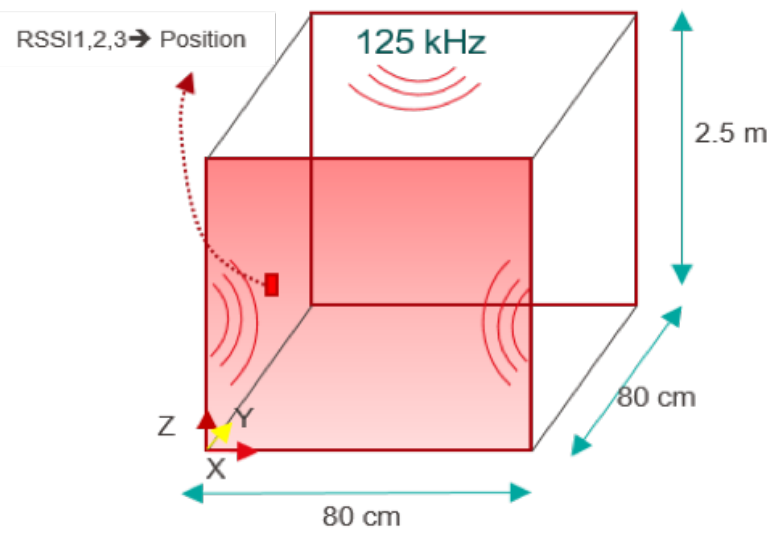

Fig. 2. Schematic of building blocks of each physical node (the receiver) in the network.

\section{POSITIONING ALGORITHM}

For real-time tests and device validation, a frame was made to fix the antennas and to accurately collect data. A frame as described in figure 2 was used to move the card within the area and measure the RSSI value in the card. The antennas were set at fixed position 3 at the height of $1 \mathrm{~m}$ above the ground.

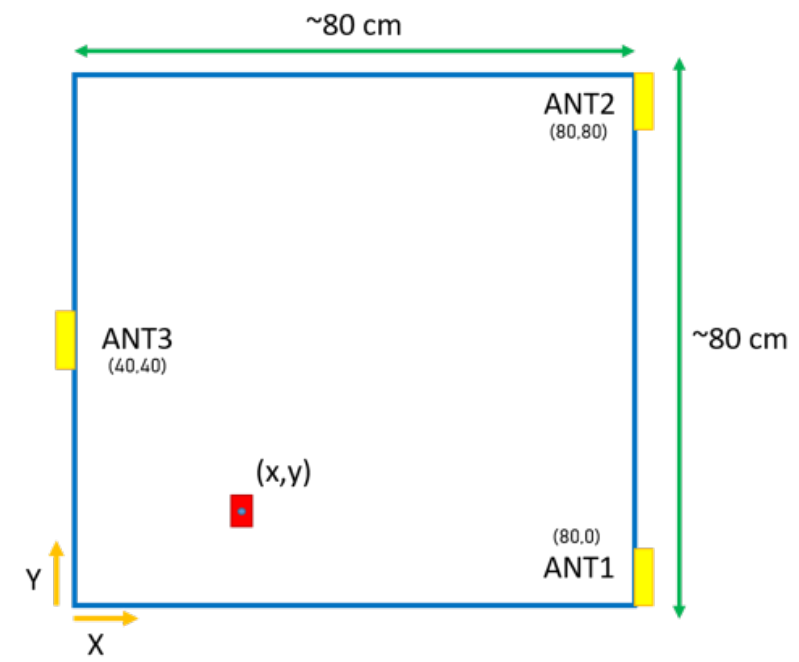

Fig. 3. A simplified $2 \mathrm{D}$ view of Antenna positions in the $80 \times 80 \mathrm{~cm}$ test area

As it was demonstrated in our last paper, the RSSI distance relation of this system obeys the following equation 
1. so that $R S S I=10 \lg \left(P_{R X}\right)$ then the measured data by the AMS3933 enables us to find a relatively linear relationship between the recorded RSSI value and the position of the card. This relationship is modeled by:

$$
R S S I=-10 n l g\left(d / d_{0}\right)+A+X_{\sigma}
$$

Where $d$ is the distance between the receiver and the transmitter, $\mathrm{n}$ is a parameter related to the specific wireless transmission environment. A is the RSSI value in the position of $d_{0}$ and the final term $\left(X_{\sigma}\right)$, is a Gaussian distributed random variable with the 0 mean and variance of $\sigma^{2}$.

Using the trilateration algorithm, and least mean square for a given node with more than 3 anchors with the coordinates of $(x 1, y 1),(x 2, y 2), \ldots(x m, y m)$ and an unknown location of a blind node we have:

$$
\left\{\begin{array}{l}
\left(x_{1}-x\right)^{2}+\left(y_{1}-y\right)^{2}=d_{1}^{2} \\
\left(x_{2}-x\right)^{2}+\left(y_{2}-y\right)^{2}=d_{2}^{2} \\
\vdots \\
\left(x_{m}-x\right)^{2}+\left(y_{m}-y\right)^{2}=d_{m}^{2}
\end{array}\right.
$$

Subtracting the $m$-th equation from the first $m-1$ equation in 2, the linear equation is obtained as $A x=b$. Where $\mathrm{A}$ and $\mathrm{b}$ are:

$$
\begin{gathered}
A=\left[\begin{array}{cc}
2\left(x_{1}-x_{m}\right) & 2\left(y_{1}-y_{m}\right) \\
2\left(x_{2}-x_{m}\right) & 2\left(y_{2}-y_{m}\right) \\
\vdots & \vdots \\
2\left(x_{m-1}-x_{m}\right) & 2\left(y_{m-1}-y_{m}\right)
\end{array}\right] \\
b=\left[\begin{array}{c}
x_{1}^{2}-x_{m}^{2}+y_{1}^{2}-y_{m}^{2}+d_{1}^{2}-d_{m}^{2} \\
x_{2}^{2}-x_{m}^{2}+y_{2}^{2}-y_{m}^{2}+d_{2}^{2}-d_{m}^{2} \\
\vdots \\
x_{m-1}^{2}-x_{m}^{2}+y_{m-1}^{2}-y_{m}^{2}+d_{m-1}^{2}-d_{m}^{2}
\end{array}\right]
\end{gathered}
$$

and Finally, by solving the equation due to linear algebra for $\mathrm{X}$ we have:

$$
X=\left(A^{T} A\right)^{-1} A^{T} b
$$

The RSSI-distance equation is inherently non-linear especially in shorter ranges (below $1 \mathrm{~m}$ ). As a result, using non-linear estimation can be considered to be an alternative solution to this problem. However, linear estimation can serve as a benchmark for further analysis. Regarding the fluctuations of the RSSI value in real-time, we propose an algorithm to estimate the position based on the recorded RSSI (figure 4).

\section{A. Neural Network Application}

The RSSI distance loss model is well known to be a nonlinear model which makes it difficult to map directly the RSSI value to the distance. In this section, the Multilayer Perceptrons (MLP) algorithm is used which is the best trade-off between accuracy and memory requirements among other types of neural networks [11], different architectures and activation functions were tested.

MLP is a feed-forward artificial neural network for supervised learning (or backpropagation training). MLP

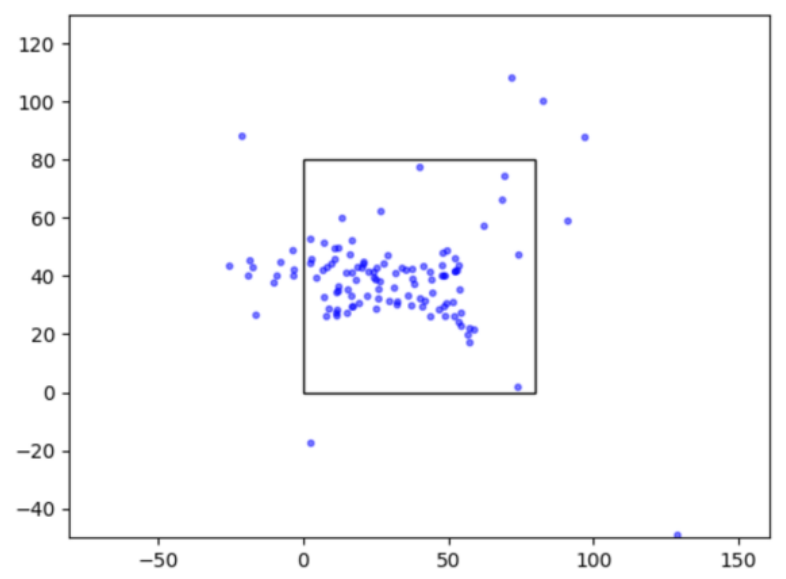

Fig. 4. The estimated position of the card in $80 \mathrm{~cm}$ by $80 \mathrm{~cm}$ frame of the test area by trilateration method composed of an input layer to receive the signal, an output layer that makes a decision or prediction about the input, and in between, an arbitrary number of hidden layers that are the true computational engine of the MLP. It is common for the performance of this type of systems to be evaluated by varying their architecture. In this work, networks with two types of activation function were evaluated, the linear function and the Elliot symmetric sigmoid function. Similarly, the number of neurons in each hidden layer varied between 25, 30, 35, 40 and the number of layers was 5, 6, and 7. In total, 24 different neural networks were trained. The purpose of the evaluation is to observe the variation of the error, not always increasing the size of the network improves its performance. In general, MLP with non-linear activation functions are expected to have minor errors, however the training time is considerably longer.

The training sample input value is $R S S I=$ $\left\{R S S I 1_{A N T 1}, R S S I 2_{A N T 1}, \ldots, R S S I 3_{A N T 3}\right\}$ plus the bias, the training sample output is $D=\left\{d_{x}, d_{y}\right\}$. As shown in the figure $5 \omega_{l k}$ is defined as a weight form the input layer to the hidden layers, while $\theta_{l k}$ is defined as the threshold value of the hidden layer and $\varpi_{i k}$ is the weight from the hidden layers to the output layer and $\psi$ is the threshold for the output. 


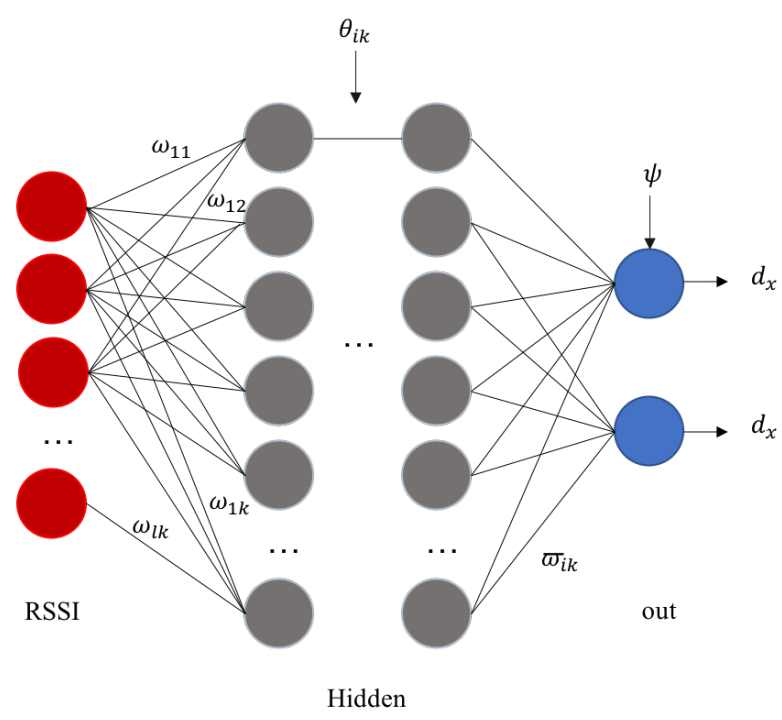

Fig. 5. Neural network structure

Figure 6, 7 presents the results of the MLP algorithm by using a linear neural function and Elliot symmetric sigmoid function. The red stars are the actual (real) position of the card. The blue stars are the estimated position by the network with an error of less than $3 \mathrm{~cm}$. It is evident that the estimation in the center of the region is better and that a greater number of samples have errors of less than $3 \mathrm{~cm}$ when the non-linear function is used than when the linear one is used.

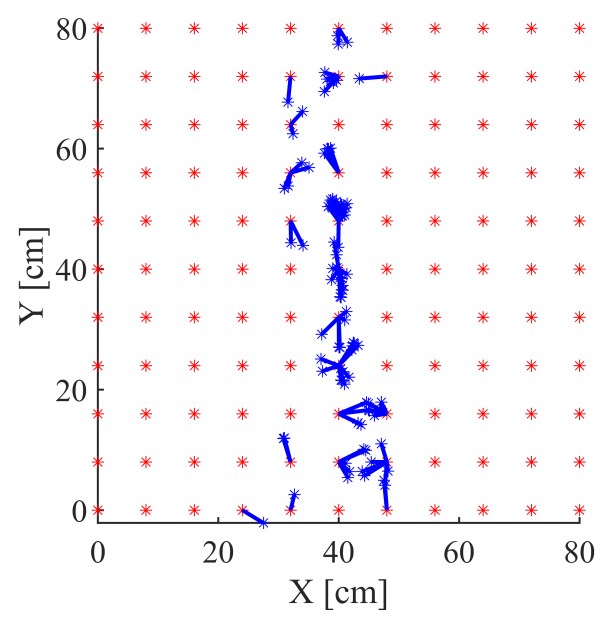

Fig. 6. Estimated position with linear function

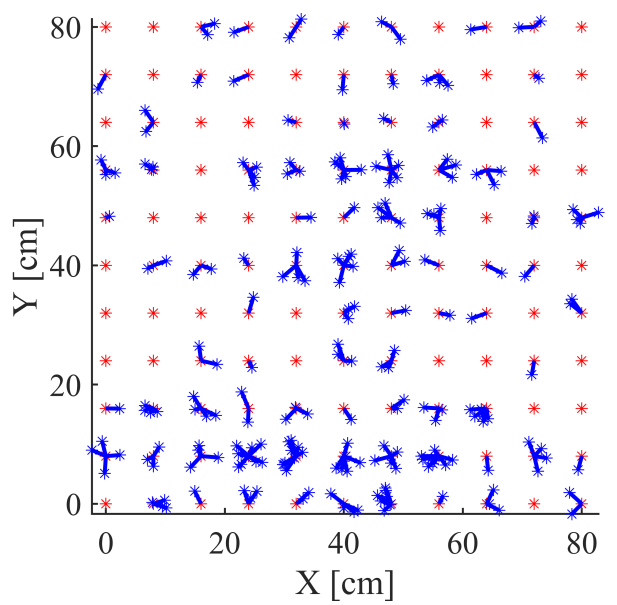

Fig. 7. Estimated position with Elliot symmetric sigmoid function

As presented in figure 6, 7 the average error for $y$ direction is less than the same in $x$ direction as a result of having twice as many antennas at $y$ direction in comparison to $x$. Sometimes the absolute error, or the mean square error does not reflect, or does not allow us to observe particular conditions. In this particular case, there are variations due to phenomena such as reflection on the walls and therefore the uncertainty is greater next to them, this problem extends to the estimation that neural networks try to make.

Finally, Table I presents the percentage of estimates within each of the ranges described in the columns for some of the architectures tested, that is, the values, 3, 5, $10,15,20$, indicate that the percentage of hit within that corresponding range. In the description of architectures, LI is linear, LX is the number of layers, and HX is the number of neurons in each hidden layer. The results are in percentage, the best result is the NL_L6_H40 network, which has 6 layers and 40 neurons in each hidden layer.

TABLE I

HITS IN SPECIFIC RANGES

\begin{tabular}{l|ccccc}
\hline \multicolumn{7}{c}{ Maximum error [cm] } \\
\hline Architecture & 3 & 5 & 10 & 15 & 20 \\
\hline LI_L7_H25 & 2.6 & 4.6 & 17.4 & 29.4 & 42.5 \\
\hline LI_L7_H30 & 2.8 & 4.7 & 18.5 & 29.1 & 42.6 \\
\hline LI_L7_H35 & 2.7 & 4.7 & 17.6 & 28.8 & 42.7 \\
\hline LI_L7_H40 & 2.9 & 4.7 & 18.6 & 29.3 & 42.4 \\
\hline NL_L6_H25 & 4.8 & 10.4 & 27.2 & 42.0 & 56.9 \\
\hline NL_L6_H30 & 5.3 & 10.9 & 30.4 & 46.3 & 58.8 \\
\hline NL_L6_H35 & 4.4 & 11.3 & 30.3 & 50.0 & 62.0 \\
\hline NL_L6_H40 & 8.3 & 21.4 & 43.6 & 57.9 & 67.7 \\
\hline
\end{tabular}

\section{CONCLUSION}

As it is presented in the 6 and 7, using a non-linear neural network to estimate the unknown position of a tag in the area can lead us to more accurate answers. Using traditional trilateration techniques with least square method will results in an average error of 27 and $30 \mathrm{~cm}$ in directions $y$ and $x$ respectively. Moreover, the absolute error of estimation for $x$ and $y$ direction for least square method is below $5 \mathrm{~cm}, 3 \mathrm{~cm}$. Note that having two antennae 
at $y$ direction is the reason for more accuracy in the $y$ direction in comparison to $x$. Using a non-linear neural network can increase the estimation percentage for error of $10 \mathrm{~cm}$ up to $60 \%$ in compression with linear network.

This study depicts the possibility of applying lowfrequency active RFID systems for short-range and semienclosed environment. Even though the higher frequency RFIDs are widely used for positioning assets in longer ranges, fewer scholars have dedicated themselves to study the effects of low-frequency active RFIDs. This frequency has the superiority of not being prone to multipath fading and improved diffraction around the corners and complex structures especially in compression to $\mathrm{MHz}$ and $\mathrm{GHz}$ frequency spectrum.

Moreover, using the intrinsic properties of this frequency, and benefiting from the magnetic coupling effect, this system can reduce the power consumption because it will go to the sleep mode if it is outside the range of the transmitter's magnetic field. This feature also creates another advantage. Because it reveals the presence of the tag as soon as it is in the area. This feature alone can be a marker for checking the absence or the presence of tags in a defined area.

\section{ACKNOWLEDGMENT}

Here, the authors would like to acknowledge our gratitude towards the contribution of our collaborators at Haute Ecole Arc Ingénierie in developing the docker engine, Professor François Tièche and his team, Nicolas Sommer and Rabia Saeed.

\section{REFERENCES}

[1] W. Sakpere, M. Adeyeye Oshin, and N. Mlitwa, "A state-ofthe-art survey of indoor positioning and navigation systems and technologies," South African Computer Journal, vol. 29, p. 145, 12 2017.

[2] P. Rong and M. L. Sichitiu, "Angle of arrival localization for wireless sensor networks," in 2006 3rd annual IEEE communications society on sensor and ad hoc communications and networks, vol. 1 , pp. 374-382, Ieee, 2006.

[3] L. Brás, N. B. Carvalho, P. Pinho, L. Kulas, and K. Nyka, "A review of antennas for indoor positioning systems," International Journal of Antennas and Propagation, vol. 2012, 2012.

[4] R. Mautz and S. Tilch, "Survey of optical indoor positioning systems," in 2011 international conference on indoor positioning and indoor navigation, pp. 1-7, IEEE, 2011.

[5] Z. Farid, R. Nordin, and M. Ismail, "Recent advances in wireless indoor localization techniques and system," Journal of Computer Networks and Communications, vol. 2013, 2013.

[6] C. L. Bethel and R. R. Murphy, "Survey of non-facial/nonverbal affective expressions for appearance-constrained robots,' IEEE Transactions on Systems, Man, and Cybernetics, Part C (Applications and Reviews), vol. 38, no. 1, pp. 83-92, 2007.

[7] D. Dardari, P. Closas, and P. M. Djurić, "Indoor tracking: Theory, methods, and technologies," IEEE Transactions on Vehicular Technology, vol. 64, no. 4, pp. 1263-1278, 2015.

[8] W. Y. Chang, H. Abu-Amara, and J. F. Sanford, Transforming enterprise cloud services. Springer Science \& Business Media, 2010.

[9] “postgresql." https://www.postgresql.org/. Accessed: 2020-8-12.

[10] "mosquitto." https://www.mosquitto.org/. Accessed: 2020-8-12.

[11] M. Abdelhadi, M. Anan, and M. Ayyash, "Efficient artificial intelligent-based localization algorithm for wireless sensor networks," Cyber Journals: Multidisciplinary Journals in Science and Technology, Journal of Selected Areas in Telecommunications (JSAT), vol. 3, pp. 10-18, 2013. 